\title{
Tbx18 regulates the development of the ureteral mesenchyme
}

\author{
Rannar Airik, Markus Bussen, Manvendra K. Singh, Marianne Petry, and Andreas Kispert \\ Institut für Molekularbiologie, Medizinische Hochschule Hannover, Hannover, Germany.
}

\begin{abstract}
Congenital malformations of the urinary tract are a major cause of renal failure in children and young adults. They are often caused by physical obstruction or by functional impairment of the peristaltic machinery of the ureter. The underlying molecular and cellular defects are, however, poorly understood. Here we present the phenotypic characterization of a new mouse model for congenital ureter malformation that revealed the molecular pathway important for the formation of the functional mesenchymal coating of the ureter. The gene encoding the T-box transcription factor $\mathrm{Tbx} 18$ was expressed in undifferentiated mesenchymal cells surrounding the distal ureter stalk. In $\mathrm{Tb} \times 18^{-/-}$mice, prospective ureteral mesenchymal cells largely dislocalized to the surface of the kidneys. The remaining ureteral mesenchymal cells showed reduced proliferation and failed to differentiate into smooth muscles, but instead became fibrous and ligamentous tissue. Absence of ureteral smooth muscles resulted in a short hydroureter and hydronephrosis at birth. Our analysis also showed that the ureteral mesenchyme derives from a distinct cell population that is separated early in kidney development from that of other mesenchymal cells of the renal system.
\end{abstract}

\section{Introduction}

Congenital anomalies of the urinary tract (ureter) constitute a major cause of chronic renal failure in children. Structural malformations of the ureter and its connection to the kidney and bladder and functional impairment of the smooth muscle (SM) layer can lead to a failure to conduct urine from the renal pelvis to the bladder. Depending on the extent of the obstruction, the accumulated urine will flow back and cause dilatation of the ureter (hydroureter) and/or the renal pelvis (hydronephrosis). Hydronephrosis can culminate in fluid pressure-mediated destruction of the renal parenchyme, ultimately necessitating renal transplantation (1-4).

Ureter development starts with the formation of the Wolffian ducts, paired epithelial tubes that extend along the anterior-posterior embryonic axis in the intermediate mesoderm. By E9.5, the Wolffian ducts have reached the primitive urogenital sinus, the primordium of the urethra, and the bladder. One day later an epithelial outgrowth, the ureteric bud, appears at the level of the future hind limbs and invades a condensation of the intermediate mesoderm, the metanephric blastema. The proximal part of the ureteric bud undergoes branching morphogenesis and differentiates into the collecting duct system, a process that depends on signals from surrounding metanephric mesenchymal cells. The distal part of the ureteric bud that lies outside the kidney elongates to form the ureter tube. The ureteric epithelium differentiates into the urothelium, which also lines the inner surface of the renal pelvis and the bladder. The surrounding mesenchymal cells eventually differentiate into an inner layer of SMCs, which functions to coordinately propel the urine downward by peristaltic movements, and an outer layer of fibrocytes $(5,6)$.

The etiology and the developmental mechanisms underlying inherited disorders of the ureter are now beginning to be unrav-

Nonstandard abbreviations used: Bmp, bone morphogenetic protein; Pax2, paired box gene 2; Pod1, podocyte expressed-1; sFRP, secreted frizzled related protein, Shh, sonic hedgehog; SM, smooth muscle; Tbx, T-box; Uncx4.1, Unc4.1 homeobox.

Conflict of interest: The authors have declared that no conflict of interest exists. Citation for this article: J. Clin. Invest. 116:663-674 (2006). doi:10.1172/JCI26027. eled $(2,3,7,8)$. Gene targeting approaches in the mouse have uncovered important regulators of ureteric epithelium development and thus of potential disease mechanisms. Budding and branching of the ureteric epithelium is triggered by glial-derived neurotrophic factor (GDNF) from the adjacent metanephric mesenchyme (9). Mutations in genes that regulate the temporal-spatial expression and action of GDNF are among the major reasons for urinary tract anomalies (3). In contrast, much less attention has been paid to the genetic control of distal ureter (stalk) development, particularly the recruitment, morphogenesis, and differentiation of the ureteral mesenchyme.

T-box $(T b x)$ genes encode a family of proteins sharing a highly conserved DNA-binding region, the T-box. They act as transcription factors that exert distinct transcriptional activation and repression functions depending on the molecular context of the conserved DNA-binding site. In mammals, 17 Tbx genes have been identified. Gene targeting experiments in mice have revealed their critical functions during gastrulation and the development of various organ systems $(10,11)$. In addition, mutations in several Tbx genes cause congenital human diseases, demonstrating the importance of the gene family both in development and disease (12).

$T b x 18$ is a member of the $T b x 15 / T b x 18 / T b x 22$ subgroup of the $T b x 1$ subfamily of $T b x$ genes. $T b x 18$ is expressed in anterior somite halves in the paraxial mesoderm, the septum transversum and the developing epicardium, the mandibular/maxillary region, the urogenital ridge, and the limb buds of gastrulation stage mouse embryos (13). Mice carrying a null allele of Tbx18 die shortly after birth due to severe malformations of the axial skeleton (14). Here we report that newborn $T b \times 18^{-/-}$mice also displayed a prominent hydroureter and hydronephrosis phenotype. We correlated expression of $T b x 18$ in the (prospective) ureteral mesenchyme with early onset of ureter abnormalities and show that $T b x 18$ regulated condensation of mesenchymal cells around the distal ureter stalk. We further demonstrate that the remaining ureteral mesenchymal cells failed to differentiate into SMCs, that both the epithelial and the mesenchymal compartments of the ureter showed reduced cell proliferation, and that the ureteric epithelium did not differenti- 

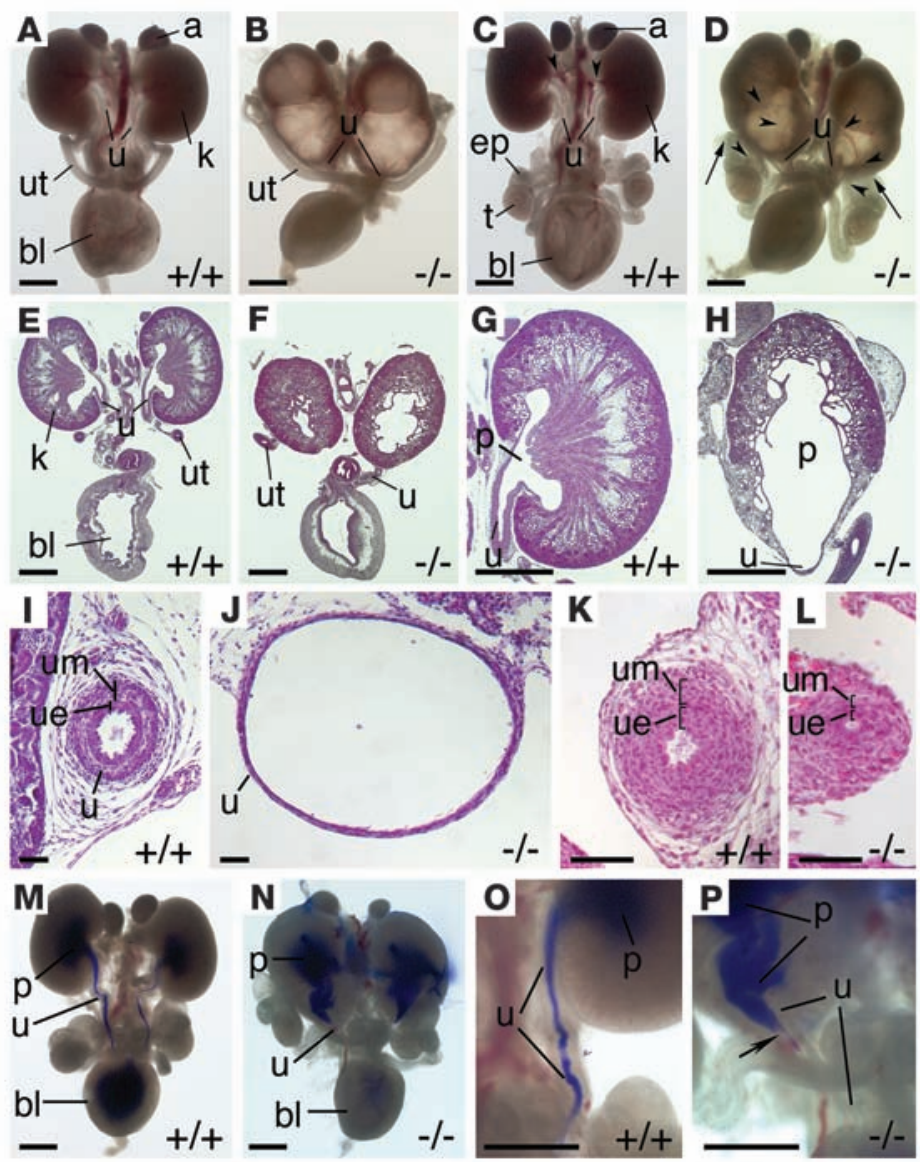

\section{Figure 1}

Kidney and urogenital tract anomalies in $\mathrm{Tb} \times 18^{-/-}$embryos at E18.5. (A-D) Morphology of whole urogenital systems of female ( $\mathbf{A}$ and $\mathbf{B}$ ) and male embryos ( $\mathbf{C}$ and $\mathbf{D}$ ). Arrowheads in $\mathbf{C}$ and $\mathbf{D}$ indicate blood vesels. Arrows in $\mathbf{D}$ indicate ligaments connecting the testes to the kidneys. (E-L) Hematoxylin and eosin stainings of sagittal sections of whole urogenital systems ( $E$ and $F$ ) and kidneys ( $\mathbf{G}$ and $\mathbf{H}$ ) and of transverse sections of the ureter (I-L). (I and $\mathbf{K}$ ) The ureter epithelium and its surrounding mesenchyme were multilayered in the wild-type embryo at the kidney level (I) and more distally (K). ( $\mathbf{J}$ and $\mathbf{L}$ ) In the $\mathrm{Tb} \times 18^{-/-}$ureter, a single-layered epithelium was surrounded by a thin layer of mesenchymal cells at proximal $(\mathbf{J})$ and distal (L) levels. (M-P) Physical obstruction of the $T b \times 18^{-/-}$ureter as revealed by ink injection experiments. ( $\mathbf{O}$ and $\mathbf{P}$ ) Higher-magnification views of the ureteropelvic region shown in $\mathbf{M}$ and $\mathbf{N}$. Arrow in $\mathbf{P}$ indicates obstruction in the ureter. a, adrenal; bl, bladder; ep, epididymis; k, kidney; $p$, pelvis; t, testis; u, ureter; ue, ureteric epithelium; um, ureteral mesenchyme; ut, uterus. Scale bars: 1,000 $\mu \mathrm{m}$ (A-H and M-P), $100 \mu \mathrm{m}(\mathbf{I}-\mathbf{L})$.

genital system of $T b \times 18^{-/-}$embryos. In the female mutant the uterus appeared stretched, and the location of the ovary was shifted ventrally (Figure 1B), probably as a consequence of the hydronephrosis phenotype and rotation of the kidney. In the male mutant, the testes and epididymes were connected to the kidneys with strong ligaments (Figure 1D, arrows). We observed ectopic blood vessels on these ligaments as well as on the ventral surface of $T b \times 18^{-/-}$kidneys (Figure 1D, arrowheads).

Histological analyses of E18.5 urogenital systems confirmed and extended these morphological findings (Figure 1, E-L). They revealed dilatation of the entire renal collectate into a functional urothelium. This analysis uncovers what is, to our knowledge, a new developmental defect leading to hydroureter and hydronephrosis. Our results also shed light on the early separation of mesenchymal cell populations in the developing kidney and its implication for ureter formation.

\section{Results}

Tbx $18^{-1-}$ mice develop perinatal congenital bydroureter and bydronephrosis. Tbx18 mutant mice die shortly after birth. Histological analysis of newborn $T b \times 18^{-/-}$pups revealed enlarged kidneys in addition to the vertebral column defects described previously (14). To characterize renal defects, we isolated whole urogenital systems at E18.5. Mutant urogenital systems displayed a prominent hydroureter and hydronephrosis phenotype, i.e., a fluid-filled dilatation of the entire renal collecting system including the ureter, the pelvis, and the collecting ducts (Figure 1). These abnormalities were fully penetrant and occurred bilaterally in both sexes (Figure 1, B and D). The bladder was unaffected, suggesting a primary hydroureter. Ureters were not only dilated (Figure 1, D and J) but also showed a severe length reduction bringing kidneys in closer proximity to the bladder (Figure 1, B, D, and F). Ureters from wild-type littermates originated medially from the pelvic region and turned perpendicularly toward the bladder, where they ended on the dorsal side (Figure $1, A$ and C). In contrast, mutant ureters were continuous with the posterior apex of the kidney, suggesting a $90^{\circ}$ rotation of the kidney and a dilatation of the pelvicaliceal space (Figure 1, B and D). The ureter orifice in the bladder was normal (data not shown). In addition to kidney and ureter defects, we noticed changes in the ing system, including the collecting ducts, calyx, pelvis, and ureter; atrophy of the papilla; and presence of cysts in the medulla in Tbx $18^{-/-}$kidneys, lesions typically associated with hydronephrosis and hydroureter (Figure 1, F, H, and J). In transverse sections of wild-type ureters, the urothelium was multilayered and was surrounded by multiple layers of mesenchymal cells (Figure 1, I and $\mathrm{K})$. This was in strong contrast to $T b \times 18^{-/-}$ureters, where the urothelium appeared flat and single layered and was surrounded by a thin layer of mesenchymal cells (Figure 1, J and L). To analyze the lumen of the urinary tract, we performed ink injection experiments (Figure 1, M-P). In the wild-type control, the blue ink injected into the pelvicaliceal space easily flowed through the urinary path to the bladder (Figure 1, M and O). In the Tbx18 ${ }^{-/-}$urogenital system, the injected ink revealed severe irregularities of the size and surface of the lumen of the pelvicaliceal space and the ureter (Figure 1, N and $\mathrm{P})$. Increased hydrostatic pressure resulted in a slow flow of the ink to the bladder in some cases ( 3 of 10 injected mutant kidneys). In all other cases the ink failed to reach the bladder. Hence, physical blockage along the ureter may at least partially contribute to or cause the hydronephrotic phenotype of the Tbx18 mutant.

Ureter defects show an early onset in Tbx18-/- embryos. To define both the onset and the progression of urogenital malformations in Tbx18 $8^{-/-}$embryos, we analyzed urogenital systems of wild-type and Tbx18 mutant embryos from E11.5 to E18.5 (Figure 2). On the morphological level, differences between wild-type and Tbx18 mutant urogenital systems were detected from E12.5 onward. In the $T b \times 18^{--}$embryo, the kidneys were rotated by $90^{\circ}$, the pelvic region appeared relocalized to the posterior apex, and the ureters 
A
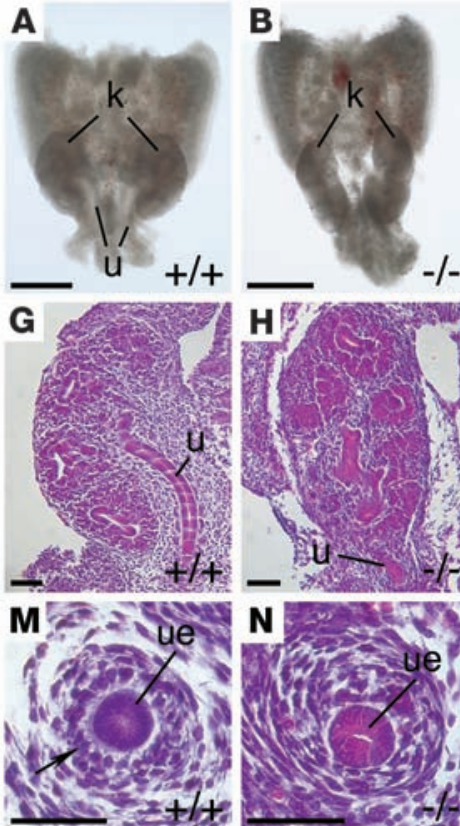

s
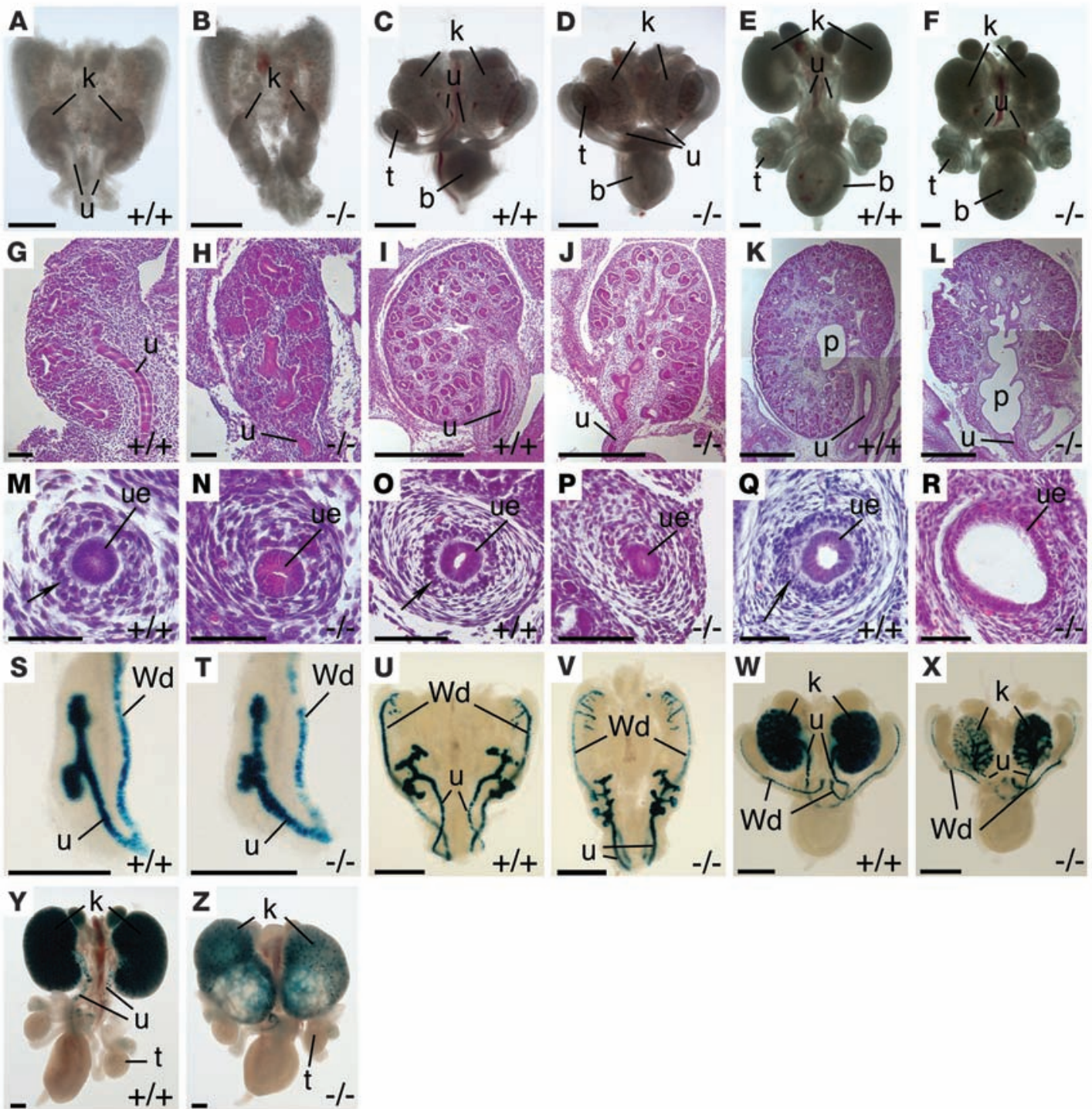

Figure 2

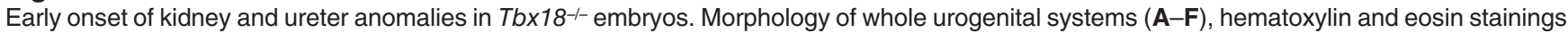
of sagittal sections of kidneys $(\mathbf{G}-\mathbf{L})$, and transverse sections of ureters $(\mathbf{M}-\mathbf{R})$ at $\mathrm{E} 12.5(\mathbf{A}, \mathbf{B}, \mathbf{G}, \mathbf{H}, \mathbf{M}$, and $\mathbf{N}), \mathbf{E} 14.5(\mathbf{C}, \mathbf{D}, \mathbf{I}, \mathbf{J}, \mathbf{O}$, and $\mathbf{P})$, and E16.5 (E, F, K, L, Q, and $\mathbf{R}$ ). Arrows in $\mathbf{M}, \mathbf{O}$, and $\mathbf{Q}$ indicate an inner ring of condensed mesenchymal cells around the ureteric epithelium that was missing in the mutant $(\mathbf{N}, \mathbf{P}$, and $\mathbf{R})$. (S-Z) $\beta$-Galactosidase activity staining of kidneys at $\mathrm{E} 11.5$ (S and $\mathbf{T})$ and of whole urogenital systems at E12.5 ( $\mathbf{U}$ and $\mathbf{V}), \mathrm{E} 14.5$ ( $\mathbf{W}$ and $\mathbf{X}$ ), and E18.5 ( $\mathbf{Y}$ and $\mathbf{Z}$ ) of a Pax2:LacZ reporter gene driving expression exclusively into the epithelium of Wolffian duct (Wd) and its derivative, the ureter. Hydroureter and hydronephrosis occurred after onset of urine formation at E16.5 (F, L, and $\mathbf{R})$ and were clearly developed by E18.5 (Z). Scale bars: $500 \mu \mathrm{m}(\mathbf{A}-\mathbf{F}, \mathbf{I}-\mathbf{L}$, and $\mathbf{S}-\mathbf{Z}), 50 \mu \mathrm{m}(\mathbf{G}, \mathbf{H}$, and $\mathbf{M}-\mathbf{R})$.

were severely shortened (Figure 2, B, D, and F). At E16.5, the failure of the testes to separate from the kidneys was apparent (Figure $2 \mathrm{~F}$ ). Histological analysis of sagittal sections of wild-type and Tbx18 mutant kidneys at E12.5, E14.5, and E16.5 confirmed the morphological findings of the renal defects (Figure 2, H, J, and L). Dilatation of the pelvicaliceal space at E16.5 indicated first hydronephrotic lesions due to onset of urine production shortly before (Figure 2L). Histological stainings of transverse ureter sections revealed onset of defects in the $T b \times 18^{-/-}$ureteral mesenchyme at E12.5. In the wild-type ureter of this stage, the inner ring of mesenchymal cells surrounding the ureteric epithelium had started to condense while the outer ring of mesenchymal cells remained loosely organized with cell bodies arranged in a tangential fashion (Figure 2M). At E14.5 and E16.5, a 2-layered appearance of the ureteral mesenchyme was clearly established (Figure 2, O and Q). In the Tbx $18^{-/-}$ureter, condensation of an inner ring of mesenchymal cells did not occur, leaving a loose mass of periureteral mesenchymal cells at all stages analyzed (Figure 2, N, P, and R). At E16.5, the ureter became severely dilated (Figure 2R). In summary, morphological and histologi$\mathrm{cal}$ analyses revealed onset of kidney and ureter anomalies in Tbx18 $18^{--}$embryos at E12.5 with progression of phenotypic severity during subsequent embryonic stages and onset of hydroureter and hydronephrosis at E16.5. 

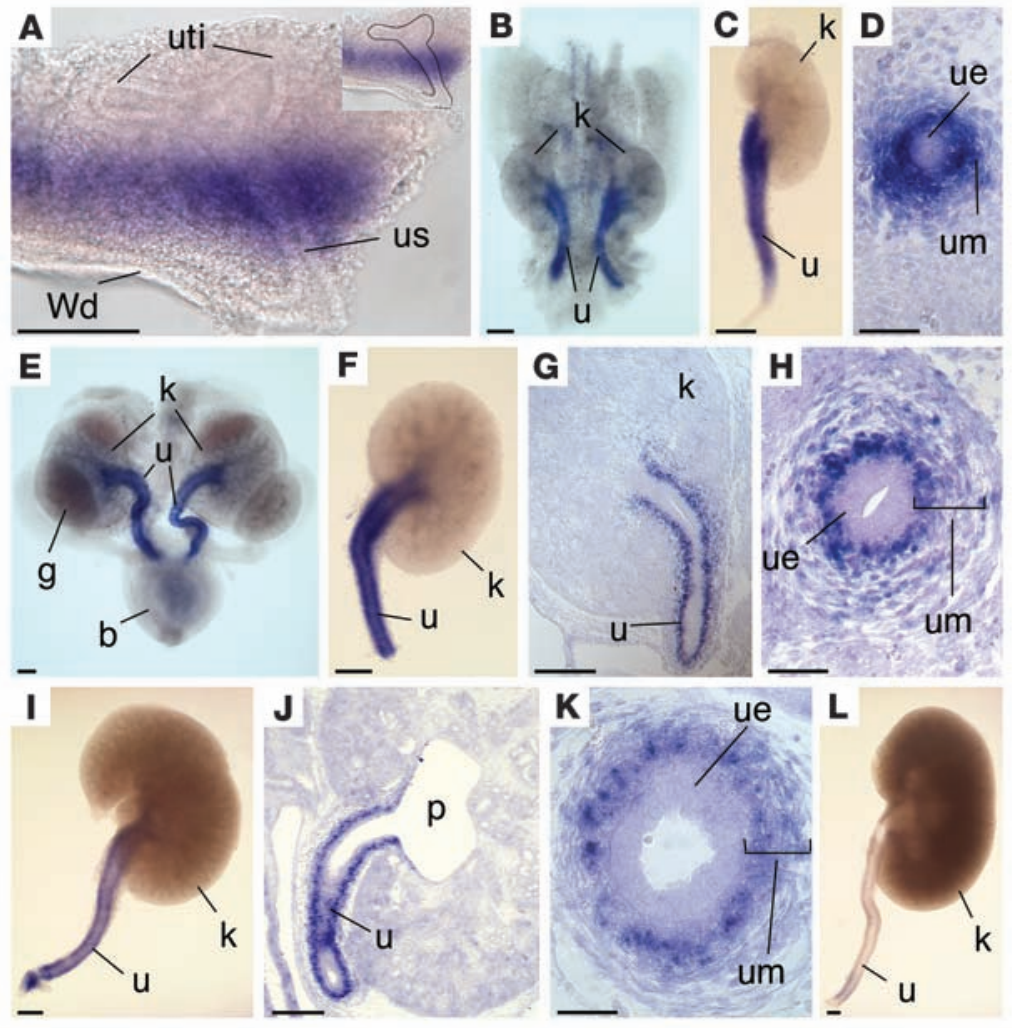

\section{Figure 3}

Tbx18 expression is confined to the developing ureter. In situ hybridization analysis of Tbx18 expression in kidneys at E11.5 (A), E12.5 (C), E14.5 (F), E16.5 (I), and E18.5 (L), in whole urogenital systems at E12.5 (B) and E14.5 (E), on transverse sections of ureters at E12.5 (D), $\mathrm{E} 14.5(\mathbf{H})$, and E16.5 (K), and on longitudinal sections of kidneys with attached ureters of wild-type embryos at $\mathrm{E} 14.5(\mathbf{G})$ and E16.5 (J). The basal surface of the ureteric epithelium is outlined (A, inset). Tbx18 expression was confined to prospective and definitive periureteral mesenchymal cells and excluded from the ureteric epithelium. Expression appeared higher in the inner layer of ureteral mesenchymal cells than in the outer ring $(\mathbf{H}$ and K). Expression was downregulated with differentiation of mesenchymal cells into SMCs after E14.5. g, gonad; uti, ureter tip; us, ureter stalk. Scale bars: $200 \mu \mathrm{m}$ (A-C, E-G, I, J, and L), $100 \mu \mathrm{m}(\mathbf{D}, \mathbf{H}$, and $\mathbf{K})$.

Tbx18 is expressed in a mesenchymal subpopulation of the developing metanephric kidney fated to form the peristaltic machinery of the ureter. Earlier work revealed expression of Tbx18 at extrarenal sites and in the urogenital ridge in E9.5-E10.5 embryos (13). However, expression during metanephric kidney development had not yet been documented. In order to address the spatial distribution of Tbx18 transcripts during kidney development, we examined Tbx18 expression in metanephric kidneys from E11.5 to E18.5 by whole-mount and section in situ hybridization analysis (Figure 3 ).

Since early ureter defects are not easily morphologically recognized, we additionally visualized the development of the Wolffian duct and its derivative, the ureter, by a transgenic reporter (Figure $2, \mathrm{~S}-Z$ ). The $L a c Z$ reporter gene was expressed under the control of an upstream region of the transcription factor paired box gene 2 (Pax2) directing expression exclusively to the Wolffian duct and its derivatives during all stages of kidney development (15). In the E11.5 wild-type kidney, the ureter budded from the Wolffian duct, invaded the metanephric blastema, and branched once (Figure 2S). The proximal ureter continued to branch, whereas the distal region, the stalk, considerably elongated between E12.5 and E18.5 (Figure 2, U, W, and Y). At E11.5, ureter development appeared normal in Tbx18 $8^{-/-}$kidneys (Figure 2T). Ectopic budding from the Wolffian duct, a frequent finding in human congenital ureter anomalies, was never observed at this or a later stage. At E12.5, proximal branching had occurred, but distal ureter elongation had progressed considerably less. As a likely consequence of the mechanical tension exerted by the shortened ureter, the kidney failed to ascend but was forced in a $90^{\circ}$ downward rotation; the pelvic region shifted posteriorly (Figure $2 \mathrm{~V}$ ). Branching morphogenesis continued normally, but the overall structure of the forming collecting duct system became asymmetric due to the malpositioning of the pelvic region. Ureter stalk elongation was further hampered. Notably, the distal ureter separated from the Wolffian duct and integrated into the dorsal epithelium of the bladder as in wild-type mice (Figure 2, W and X). The reduced number of collecting ducts seen in the E18.5 mutant kidney most likely reflected the parenchymal atrophy due to hydronephrosis (Figure $2 \mathrm{Z}$ ). Thus, Pax2 reporter gene analysis during kidney and urinary tract development suggests that loss of Tbx18 primarily affects elongation of the distal ureter stalk starting at E12.5.
At E11.5, expression of Tbx18 was detected in a band of mesenchyme flanking the metanephrogenic mesenchyme on the medial side and a thin stripe of mesenchyme surrounding the Wolffian duct on the lateral side. Expression extended anteriorly into the mesonephric region (Figure 3A). At E12.5, Tbx18 expression was found in the distal ureter stalk but not in the pelvic region, the collecting duct system, or the urogenital sinus (Figure 3, B and C). Expression remained confined to the distal ureter stalk during further development (Figure 3, E-G, I, and J). It was high until E14.5 but was downregulated concomitantly with the proximodistal wave of SM differentiation. In situ hybridization analysis of whole urogenital systems at E12.5 and E14.5 confirmed that Tbx18 expression was confined to the ureter and excluded from all other components of the urogenital system including the bladder, gonads, Wolffian duct, and Müllerian duct (Figure 3, B and E). Section in situ hybridization analysis revealed restriction of $T b \times 18$ expression to the mesenchymal cell layer surrounding the (distal) in the inner layer of mesenchymal cells than in the outer layer, however, a finding that may reflect higher cell density rather than increased expression per cell in this region (Figure 3, D, H, and K). This analysis suggests that the ureteral mesenchymal cell lineage is separated early from other mesenchymal cell lineages contributing to metanephric development. Tbx18 expression correlates with the undifferentiated state of periureteral mesenchymal cells.

Tbx18-expressing cells are mislocalized in Tbx18-/- kidneys. Tbx18 is expressed in mesenchymal cells of the ureter, suggesting that the arrest of elongation of the ureteric epithelium may be secondary to changes in the surrounding mesenchymal cell population. To trace the fate of $T b x 18$-expressing cells in the developing urogenital systems of $T b \times 18^{+/-}$and $T b \times 18^{-/-}$embryos, we took advantage ureteric epithelium from E12.5 on. Expression appeared higher 
A
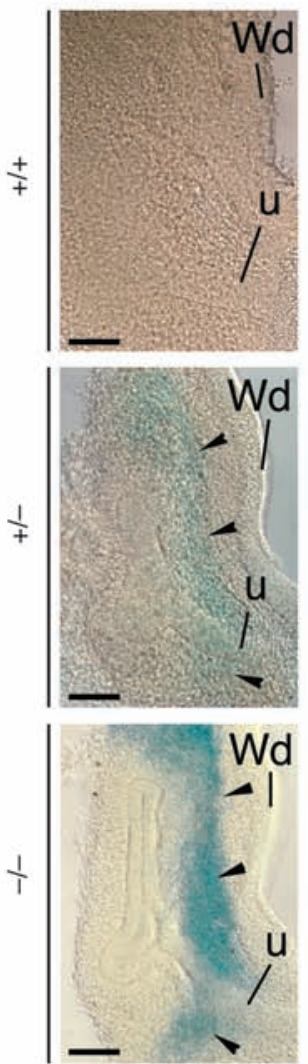

$\mathbf{F}$
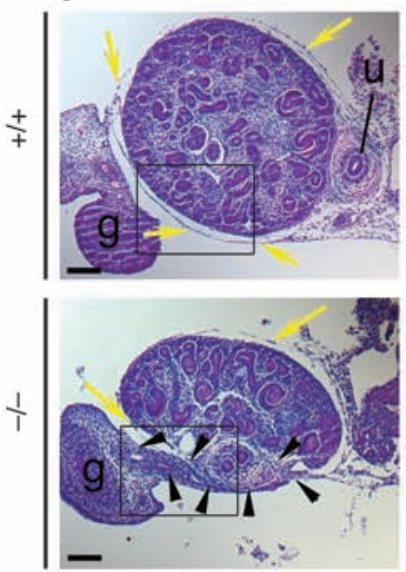

B
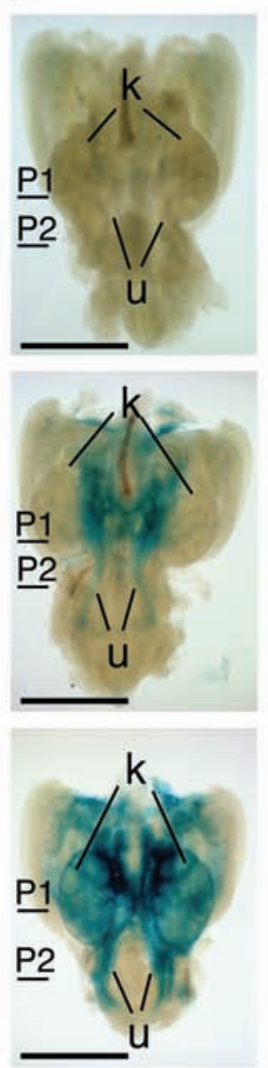

G
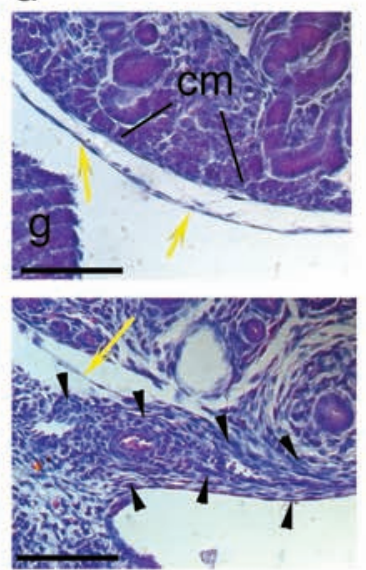

C
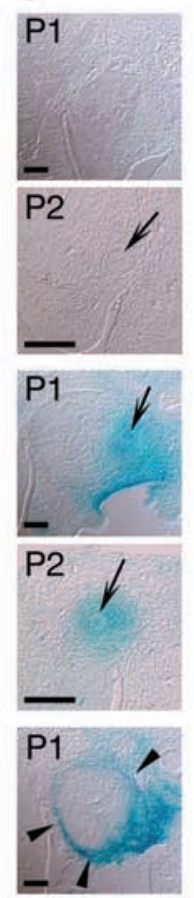

P2

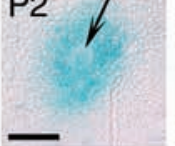

D
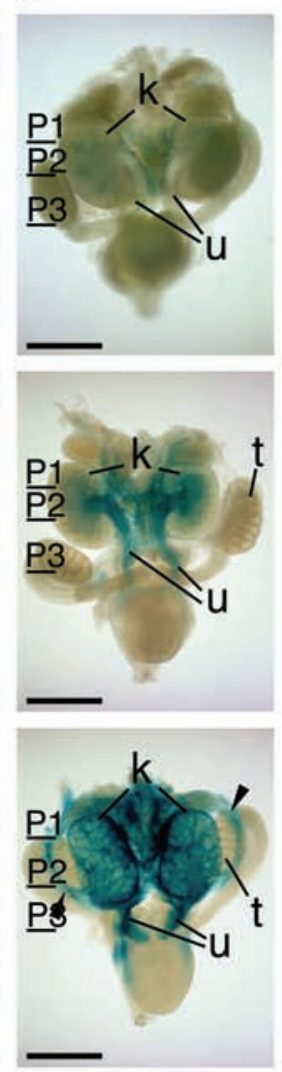

E
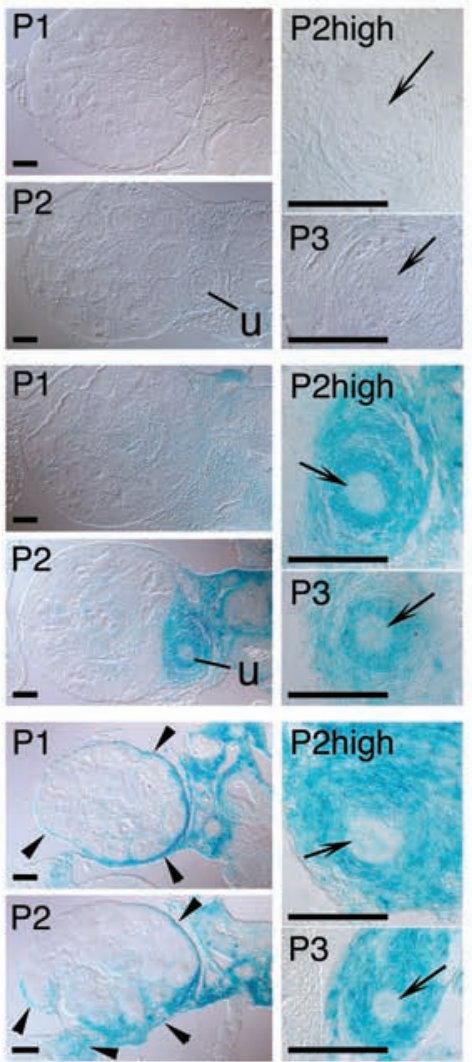

\section{H}
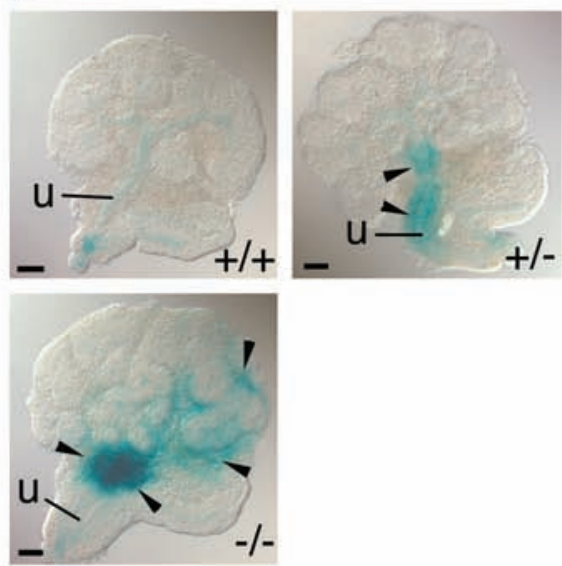

\section{Figure 4}

Mislocalization of Tbx18:LacZ expression in Tbx18 ${ }^{-/-}$urogenital systems. (A-E) $\beta$-Galactosidase activity staining of a $L a c Z$ reporter gene in the Tbx18 locus was analyzed in kidneys at E11.5 (A), in urogenital systems at E12.5 (B) and E14.5 (D), and in transverse sections of E12.5 (C) and E14.5 kidneys (E). P1 and P2 in C indicate sections from the planes shown in B. P1, P2, and P3 in E indicate section planes shown in $\mathbf{D}$. P2high is a higher magnification of $\mathrm{P} 2$ in the region of the ureter. Black arrows indicate the ureteric epithelium. (F and G) Hematoxylin and eosin stainings of transverse sections of E14.5 kidneys. Yellow arrows indicate the renal capsule. The boxed regions in $\mathbf{F}$ are magnified in $\mathbf{G}$. $(\mathbf{H}) \beta$-Galactosidase activity staining of 2-day-old cultures of metanephric rudiments that were wild type as well as heterozygous and homozygous for the mutant $T b x 18^{\mathrm{Lac} Z}$ allele. Slight blue staining of the ureteric epithelium in wild-type mice was due to endogenous $\beta$-galactosidase activity. Heterozygotes and homozygotes in A, B, and D were Tbx18 LacZ/+ and Tbx18 LacZ/Tbx18 LacZ, respectively, whereas embryos used for $\mathbf{C}$ and $\mathbf{E}$ were normalized for the $L a c Z$ allele (i.e., $T b x 18^{L a c Z} /+$ and $T b x 18 / T b x 18^{L a c z}$, respectively). $\mathbf{c m}$, condensing (metanephric) mesenchyme. Arrowheads in A and $\mathbf{C}-\mathbf{H}$ mark localization of Tbx18-positive prospective and definitive ureteral mesenchymal cells in kidneys, urogenital systems, and kidney cultures at the indicated stages. Scale bars: $100 \mu \mathrm{m}(\mathbf{A}, \mathbf{C}$, and $\mathbf{E}-\mathbf{H}), 1,000 \mu \mathrm{m}$ (B and D). 

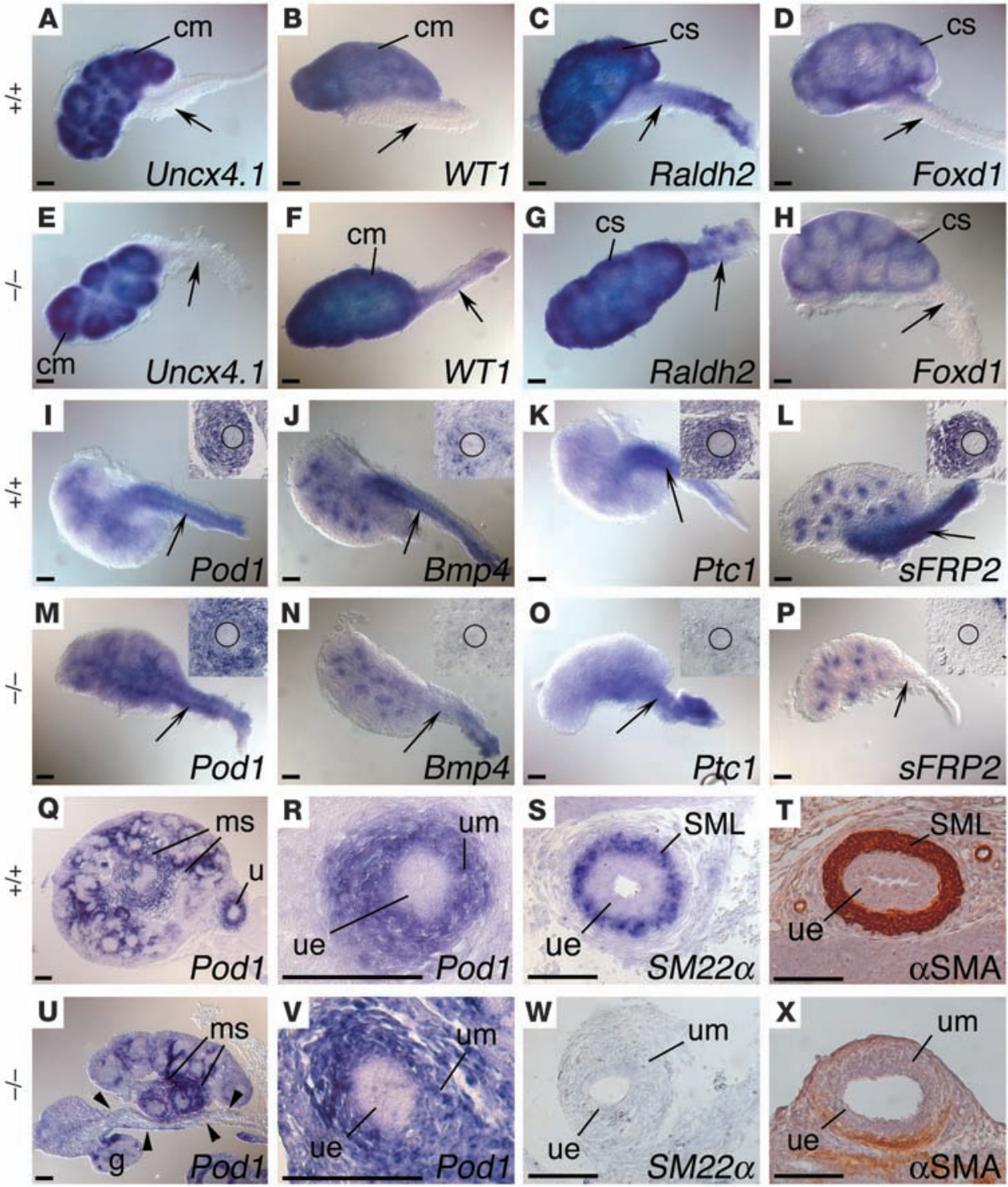
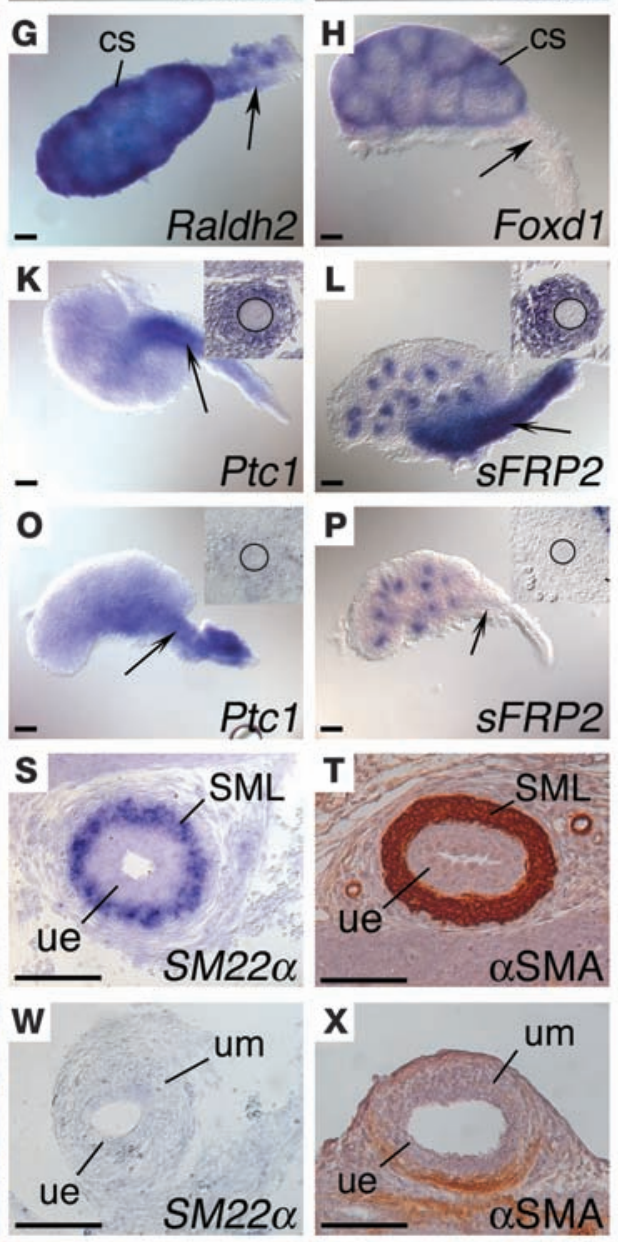
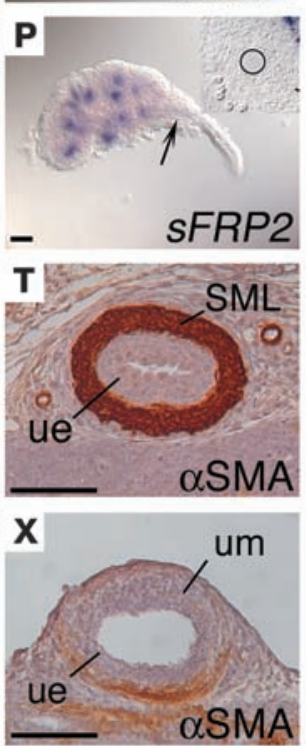

\section{Figure 5}

Molecular characterization of Tbx18--- ureteral mesenchyme. Whole-mount in situ hybridization analysis of mesenchymal marker genes in E12.5 kidneys and ureters $(\mathbf{A}-\mathbf{P})$. Arrows indicate ureteral mesenchyme. Insets in (I-P) show in situ hybridization analysis on transverse ureter sections. Ptc1, Patched1. The ureteric epithelium is shown by a black circle. In situ hybridization analysis of Pod1 expression on transverse sections of E14.5 kidneys ( $Q$ and $\mathbf{U}$ ) and ureters (R and $\mathbf{V})$ and of SM22 $\alpha$ expression on transverse sections of E16.5 ureters (S and W). Arrowheads in $\mathbf{U}$ indicate ectopic fibrous tissue connecting gonad and kidney. ( $\mathbf{T}$ and $\mathbf{X}$ ) Immunohistochemistry for $\alpha$-SMA on transverse sections of E18.5 ureters. cs, cortical stroma; ms, medullary stroma; SML, smooth muscle layer. Scale bars: $100 \mu \mathrm{m}$, $120 \mu \mathrm{m}$ (insets). of a $L a c Z$ reporter gene integrated in the Tbx18 locus (14). $\beta$-Galactosidase activity staining in the urogenital system of heterozygous Tbx18 mice from E11.5 to E18.5 faithfully reflected the expression of Tbx18 mRNA, i.e., the $\beta$-galactosidase activity was restricted to prospective and definitive ureteral mesenchymal cells (Figure 4, $\mathrm{A}-\mathrm{E}$, middle panels). Taking advantage of the stability of $\beta$-galactosidase protein that allows its use as a short-term lineage marker, this also showed that the band of Tbx18-positive mesenchymal cells in the E11.5 kidney indeed exclusively represented prospective periureteral cells, i.e., cells destined to surround the distal ureter stalk. In E11.5 Tbx $18^{-/-}$kidney rudiments, $\beta$-galactosidase activity staining was found in a broad band underlying the metanephric mesenchyme. The LacZ expression domain was unchanged compared with that of the Tbx18 heterozygous kidney, but staining intensity was stronger due to the presence of $2 \mathrm{LacZ}$ alleles (Figure $4 \mathrm{~A}$, arrowheads in bottom panel). In E12.5 $\mathrm{Tb} \times 18^{-/-}$urogenital systems, $L a c Z$ staining surrounded the short distal ureter stalk. However, the staining extended anteriorly and covered the surface of the kidneys as well (Figure 4, B and C, bottom panels). In E14.5
Tbx $18^{-/-}$urogenital systems, $\beta$-galactosidase activity staining was maintained in mesenchymal cells of the short distal ureter stalk and in a thin sheet of cells covering most of the ventral kidney surface (Figure 4, D and E, bottom panels). Some LacZ staining was also located in ligaments connecting the testes to the kidneys (Figure 4D, arrowheads). The $\beta$-galactosidase activity staining became weaker after E14.5 and was hardly detected at E18.5, both in the heterozygous and homozygous Tbx18 urogenital systems, in agreement with the downregulation of $T b \times 18$ expression at these stages (data not shown).

In summary, in $T b \times 18^{-/-}$urogenital systems $T b \times 18$ expression is found ectopically in cells covering the surface of the kidneys in addition to ligaments tethering the gonads to the kidneys. Histological stainings of transverse sections of E14.5 Tbx18-/- kidneys revealed disruption of the kidney capsule on the ventral side and the presence of an ectopic band of fibrocytes between the gonad and the kidney (Figure 4, F and G, arrowheads). This finding argues against a transcriptional derepression of the Tbx18 locus in the $T b \times 18^{-/-}$embryo but suggests that $T b \times 18^{-/-}$prospective ureteral 

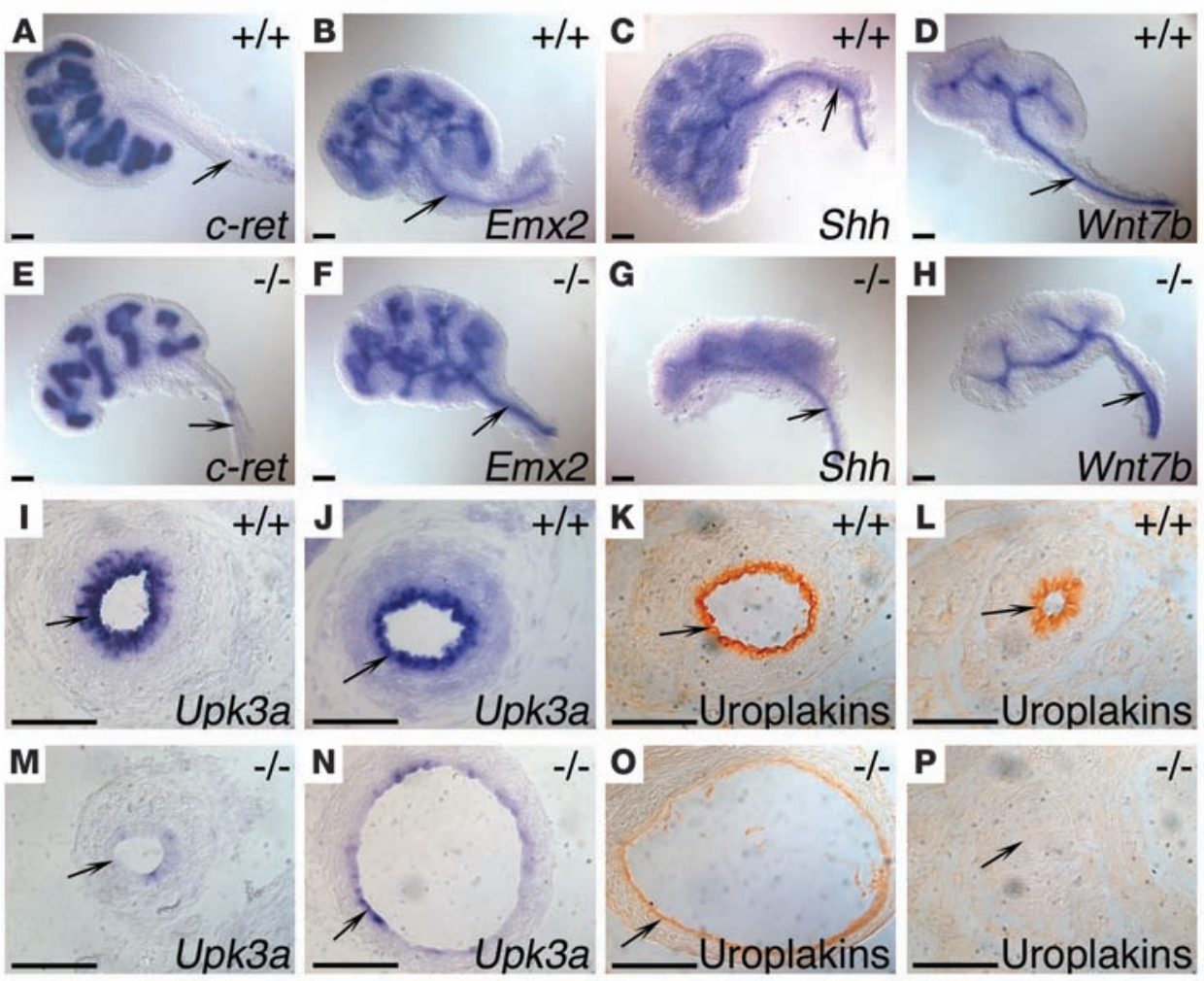

\section{Figure 6}

Molecular characterization of the Tbx $18^{-/-}$ureteric epithelium. (A-H) Whole-mount in situ hybridization analysis at E12.5. (I, J, M, and $\mathbf{N}$ ) In situ hybridization analysis on transverse ureter sections at E16.5 (I and $\mathbf{M})$ and E18.5 ( $\mathbf{J}$ and $\mathbf{N}$ ) and (K, L, $\mathbf{O}$, and $\mathbf{P}$ ) immunohistochemistry on transverse sections of E18.5 ureters at a proximal position ( $\mathbf{K}$ and $\mathbf{O}$ ) and more distally ( $\mathbf{L}$ and $\mathbf{P}$ ) of $\mathrm{Tb} \times 18^{-/-}$ embryos and control wild-type littermates. Arrows indicate ureteric epithelium. Scale bars: $100 \mu \mathrm{m}$. cells fail to condense around the ureteric epithelium and dislocalize to the surface of the kidneys closest to their initial location in the E11.5 metanephric rudiment.

Tbx18 is also expressed in the mesonephric mesenchyme (13). In order to exclude that ectopic LacZ expression was due to cells of mesonephric origin, we cultured metanephric rudiments from heterozygous and homozygous Tbx18 mutant embryos at E11.5. In 2-day-old cultures of heterozygous tissues, LacZ expression was localized to the ureter stalk (Figure $4 \mathrm{H}$, arrowheads in top right panel). In homozygous explants LacZ-positive cells remained uncondensed and partially covered the surface of the culture (Figure $4 \mathrm{H}$, arrowheads in bottom panel), ruling out the possibility that cells of mesonephric origin are the source of ectopically localized LacZ expressing cells. This also corroborates the in vivo observation that $T b \times 18^{--}$prospective ureteral mesenchymal cells failed to condense around the epithelium of the distal ureter stalk. Failure of the ureteric epithelium to grow may be a consequence of a lack of or a severe reduction in the surrounding mesenchymal cell layer.

Ureteral mesenchymal cells fail to differentiate normally in $\mathrm{Tb} \times 18^{-/-}$ureters. To reveal the underlying molecular changes in $T b \times 18^{-/-}$ureteral mesenchyme, we analyzed the expression of genes marking different types of mesenchymal cells in E12.5 kidneys and ureters (Figure 5). The paired-type Unc4.1 homeobox (Uncx4.1) transcription factor gene is expressed in the condensing metanephric mesenchyme (16), Wilm's tumor 1 (WT1) in the undifferentiated metanephric mesenchyme (17). Retinaldebyde debydrogenase (Raldh2) and Foxd1 (formerly BF2) expression is confined to the cortical stroma of the metanephros, with weak additional expression in the fibrous cell layer in the periphery of the ureteral mesenchyme (18-20). Expression of these genes was unchanged in $T b \times 18^{-/-}$kidneys (Figure 5, $\mathrm{E}-\mathrm{H})$, suggesting that loss of Tbx18 in the ureteral mesenchyme does not disturb the differentiation of other mesenchymal cell lineages in the metanephric kidney.

Podocyte expressed-1 (Pod1, also known as capsulin, epicardin), the gene encoding a basic helix-loop-helix protein, is expressed in the medullary stroma of the kidney and in the ureteral mesenchyme $(20,21)$. Expression of Pod 1 was not altered in the Tbx18 mutant kidney (Figure 5M). In contrast, expression of other genes confined to the ureteral mesenchyme was severely affected (22-25). Expression of the gene encoding Patched1 (Ptc1), a receptor for hedgehog proteins, was downregulated (Figure 5O), and bone morphogenetic protein 4 (Bmp4) and secreted frizzled related protein (Sfrp2) expression was completely lost from the ureteral mesenchyme (Figure 5, N and P). Intriguingly, Sfrp2 expression was already absent from the band of prospective ureteral mesenchymal cells underlying the metanephric mesenchyme at E11.5 (data not shown). This shows that $T b x 18$ is required for expression of specific genes in the ureteral mesenchyme. Loss of Tbx18 may affect several signaling pathways including BMP, sonic hedgehog (Shh), and that of the Wnt family of secreted glycoproteins.

Pod1 expression was found in $T b \times 18^{-/-}$mesenchymal cells around the ureter (Figure $5 \mathrm{~V}$ ), but not in ectopic cells on the kidney surface, at E14.5 (Figure 5U, arrowheads), suggesting that signals from the ureteric epithelium are required to maintain Pod1 expression.

Cells from the inner ring of ureteral mesenchymal cells differentiate into SMCs in a proximal to distal wave starting at E15.5 (22). At E16.5, expression of the SMC marker SM22 $\alpha$ indicated differentiation of SMCs in the wild-type ureter (Figure 5S). At E18.5, we detected a thick layer of SMCs surrounding the urothelium at all levels of the wild-type ureter analyzed, as judged by immunohistochemical detection of $\alpha$-SMA (Figure 5T and data not shown). In contrast, no $S M 22 \alpha$ expression and very weak 

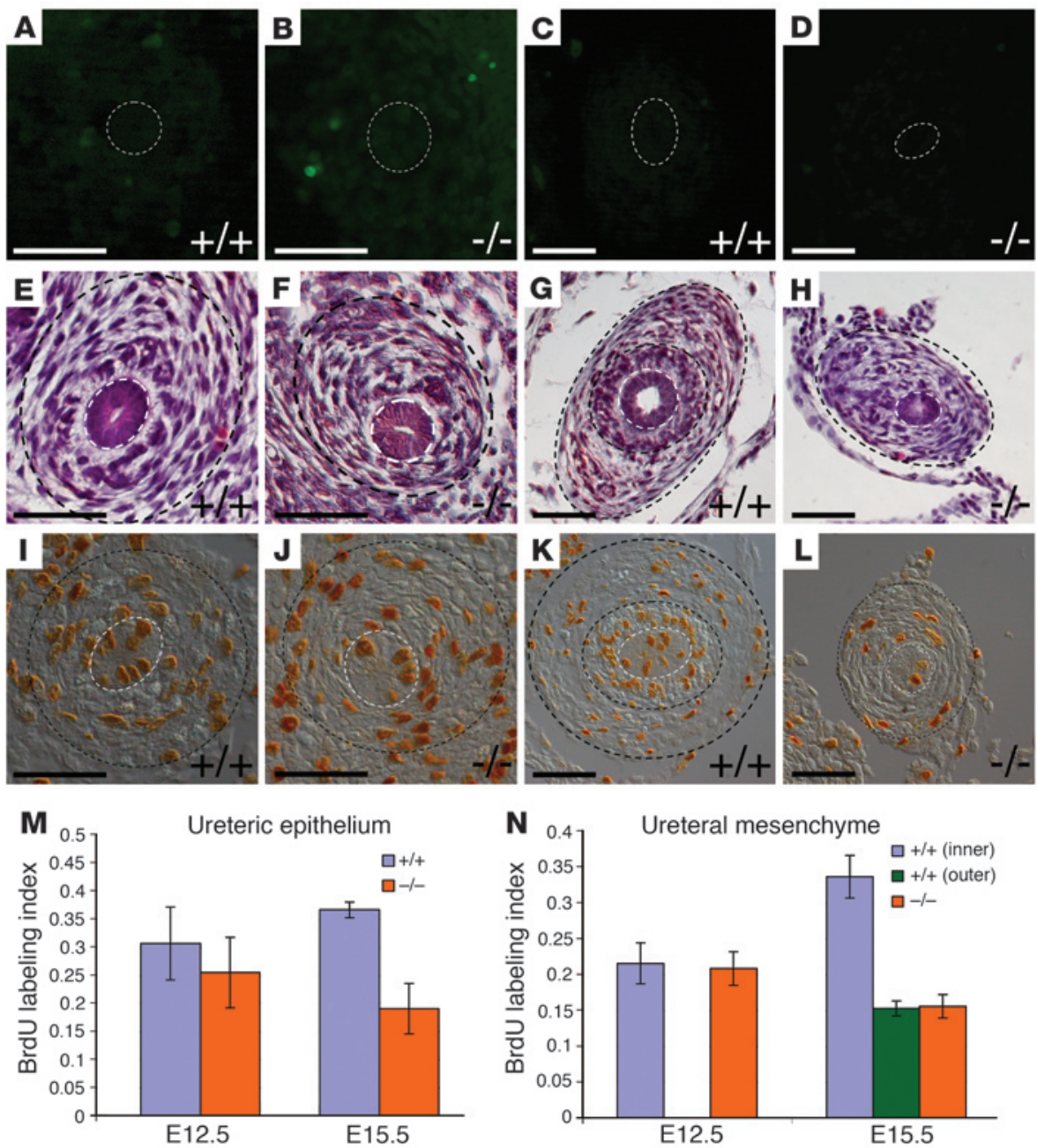

Figure 7

Apoptosis and proliferation in Tbx $18^{-/-}$ureters. (A-D) TUNEL assay on transverse sections of E12.5 (A and B) and E15.5 (C and D) ureters did not reveal a difference in apoptosis between ureteric epithelium (within dashed outline) and the ureteral mesenchyme (outside dashed outline) of Tbx18 mutant embryos (B and $\mathbf{D}$ ) and wild-type littermates (A and $\mathbf{C})$. (E-H) Hematoxylin and eosin stainings of transverse sections of wild-type and $T b \times 18^{-/-}$ureters at E12.5 (E and $\mathbf{F}$ ) and E15.5 ( $\mathbf{G}$ and $\mathbf{H}$ ). (I-L) Analysis of cell proliferation in transverse sections of the ureter of wild-type (I and $\mathbf{K}$ ) and Tbx18 mutant embryos ( $\mathbf{J}$ and $\mathbf{L}$ ) at E12.5 (I and $\mathbf{J}$ ) and E15.5 (K and $\mathbf{L}$ ) by the BrdU incorporation assay. In E-L, dashed white outlines show the ureteric epithelium and dashed black outlines the inner and outer layers of the ureteral mesenchyme as determined by the border of highly condensed mesenchymal cells and tangentially oriented loose mesenchymal cells, respectively. ( $\mathbf{M}$ and $\mathbf{N}$ ) Quantification of cell proliferation by the BrdU labeling index at E12.5 and E15.5 in the analyzed ureteral mesenchyme $(\mathbf{N})$ and in the ureteric epithelium (M). In E12.5 epithelium, $P=0.30$, wild-type $(0.31 \pm 0.07)$ versus mutant $(0.25 \pm 0.06)$. In E12.5 mesenchyme, $P=0.71$, wild-type $(0.21 \pm 0.03)$ versus mutant $(0.21 \pm 0.02)$. In E15.5 epithelium, $P<0.0003$, wild-type $(0.37 \pm 0.01)$ versus mutant $(0.19 \pm 0.04)$. In E15.5 mesenchyme, $P<0.00004$, wild-type inner ring $(0.34 \pm 0.03)$ versus mutant $(0.16 \pm 0.02) ; P=0.77$, wild-type outer ring $(0.15 \pm 0.01)$ versus mutant $(0.16 \pm 0.02)$. Scale bars: $50 \mu \mathrm{m}$.

expression of $\alpha$-SMA, respectively, were detected in mesenchymal cells surrounding the mutant ureter and $T b \times 18^{-/-}$cells ectopically localized to the kidney surface (Figure $5, \mathrm{~W}$ and $\mathrm{X}$, and data not shown). This observation suggests that $T b \times 18^{-/-}$mesenchymal cells fail to differentiate into SMCs in the developing ureter. Lack of SM layers will largely contribute to hydroureter and subsequent hydronephrosis in the $T b \times 18^{-/-}$embryo.

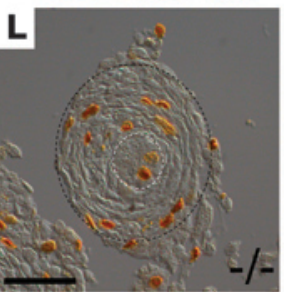

The epithelium fails to differentiate into a functional urothelium in Tbx18-/- ureters. Reciprocal signaling between adjacent tissue compartments is a hallmark of developing vertebrate organs including the metanephric kidney. To investigate whether loss of $T b \times 18$ in the ureteral mesenchyme directly leads to molecular changes in the adjacent ureteric epithelium at early stages, we studied expression of genes differentially expressed along the ureteric epithelium at E12.5 and E15.5 (Figure 6). c-ret encodes a receptor tyrosine kinase that is strongly expressed in ureter tips, weakly expressed in the (forming) collecting ducts, and absent from the distal stalk (26). Emx2 is expressed throughout the ureteric epithelium at E12.5 but is restricted to proximal regions at E15.5 (27). Wnt7b, which encodes a member of the Wnt family of signaling proteins, is expressed in the collecting ducts and in the ureter stalk at both time points (28). Shb is extremely weakly expressed in a pattern similar to that of $W n t 7 b$ (22). Expression of c-ret, $E m \times 2, W n t 7 b$, and $S h b$ was unaltered in the $T b \times 18^{-/-}$ureteric epithelium at E12.5 and E15.5 (Figure 6, E-H, arrows, and data not shown).

We wondered whether loss of Tbx 18 in the ureteral mesenchyme would subsequently compromise differentiation of the ureteric epithelium into a functional urothelium. Since urothelium is characterized by 2-dimensional crystals of uroplakins on the apical surface of the mouse urothelium (29), we analyzed uroplakin expression in E16.5 and E18.5 Tbx18 ${ }^{-/-}$ureters. In wild-type mice, Upk3a was strongly expressed in the ureteric epithelium from E16.5 onward (Figure 6, I and J). Uroplakin protein lined the inner surface of the lumen along the entire extension of the ureter at E18.5 (30) (Figure 6, K and L). In the Tbx $18^{-/-}$ureter, expression of Upk3a was weak and patchy (Figure 6, M and N). Similarly, uroplakin protein levels were low proximally and virtually absent distally (Figure 6, O and P). Hence loss of Tbx18 in the ureteral mesenchyme does not primarily affect gene expression in the undifferentiated ureteric epithelium, but prevents later differentiation into a functional urothelium. This may indicate a requirement for mesenchymal-epithelial signaling, possibly from the SMC layer to the ureteric epithelium.

Proliferation is reduced in the Tbx $18^{-/-}$ureter. We next addressed whether impairment of ureter elongation may be caused by reduced cell proliferation and/or increased apoptosis. TUNEL 
analysis in wild-type and mutant ureters did not reveal any differences in apoptosis at E12.5 and E15.5 (Figure 7, A-D; histological stainings of transverse ureter sections at the same stages are shown in Figure 7, E-H). In addition, we detected no increase in programmed cell death in transverse sections of the kidney at these stages (data not shown).

Cellular proliferation was determined by analysis of BrdU incorporation in E12.5 and E15.5 wild-type and Tbx18 mutant ureters (Figure 7, I-N). We quantitated cell proliferation in the ureteric epithelium and the ureteral mesenchyme separately to investigate whether loss of Tbx18 in the mesenchyme might - secondarily affect cell proliferation in the adjacent epithelial compartment. In addition, we separately determined BrdU indices of the inner layer of highly condensed ureteral mesenchymal cells and the outer layer of loosely organized mesenchymal cells in the E15.5 wild-type ureter and compared them with that of the homogenous layer of loosely organized ureteral mesenchyme of the $T b \times 18^{-/-}$embryo at the same stage (Figure 7, G, H, K, and L). Proliferation was insignificantly reduced in either ureter compartment at E12.5. In contrast, proliferation was significantly reduced in both compartments at E15.5, suggesting that arrest of ureter elongation in the Tbx $18^{-/-}$ embryos is accompanied and partly caused by a reduction of cellular proliferation rates (Figure 7, M and N). Intriguingly, proliferation rates of the epithelial and mesenchymal compartments were found to be similar to wild-type, albeit at a lower level. Notably, the outer layer of wild-type mesenchymal cells exhibited the same proliferation rate as the ureteral mesenchyme of the mutant mice. Reduction of proliferation rates in both compartments suggests mesenchymal-epithelial signaling, i.e., loss of Tbx18 in the ureteral mesenchyme indirectly affects epithelial cell function. This might be partly due to the failure to establish the inner ring of highly condensed, highly proliferative mesenchymal cells.

\section{Discussion}

The development of the distal ureter is a poorly understood process. Few molecular factors have been implicated in its growth and maturation, and the signaling pathways regulating the interaction between its epithelial and mesenchymal compartments are largely unknown. In this study, we examined the role of the T-box transcription factor gene Tbx18 in urinary tract development. We show that $T b \times 18$ was required for the condensation of ureteral mesenchymal cells around the distal ureteric epithelium and for the further proliferation and differentiation of these precursor cells into SMCs. The ureteric epithelium failed to differentiate into a functional urothelium. Tbx18 mutant mice developed anomalies of the urinary tract, including hydroureter and hydronephrosis, reminiscent of congenital human birth defects.

Tbx18 and specification of ureteral mesenchyme. Our study shows that the loss of Tbx18 affected the development of the mesenchymal coating of the ureter at several points. Prospective ureteral mesenchymal cells largely failed to condense around the distal ureteric epithelium. The remaining cells showed reduced proliferation and failed to form the inner SM layer, resulting in a short ureter that did not support the transport of urine to the bladder. We suggest that the effect of Tbx18 on proliferation and differentiation is secondary to its role in specifying mesenchymal precursors to become ureteral mesenchyme.

To date, the developmental origin of the mesenchyme ensheathing the ureter was not known. Our study strongly suggests that mesenchyme fated to surround the distal ureter epithelium becomes molecularly distinct as early as E11-E11.5 in kidney development. Tbx18 expression marks a band of mesenchyme adjacent to the metanephric mesenchyme on one hand and mesenchyme surrounding the epithelium of the Wolffian duct on the other. Although we did not firmly prove that these cells exclusively and definitely provide the mesenchymal cell layer of the distal ureter, it seems very likely that the 2 cell populations are indeed continuous. $\beta$-Galactosidase assays of the Tbx18:LacZ reporter gene, which serves as a short-term lineage marker, showed that all Tbx18-positive cells rapidly condensed around the distal ureter.

Notably, the Tbx18 expression domain abuts the metanephric mesenchyme that is marked by Uncx4.1 expression (16). This situation is somewhat reminiscent of the complementary expression of Tbx 18 and Uncx4.1 in anterior and posterior somite halves, respectively. There both genes coordinately maintain anterior-posterior compartmentalization $(14,31,32)$. In the somitic mesoderm loss of Tbx18 led to reduction of anterior somite halves, whereas the Tbx18 domain expanded in kidney development. Our preliminary phenotypic characterization of Uncx4.1 mutant kidneys (H. Farin and A. Kispert, unpublished observations) does not support a role for Uncx4.1 in maintaining the separation of metanephric and ureteral mesenchymal cells either, arguing for disparate molecular programs regulated by either gene in somite and kidney development.

Although the mesenchymal coating of the collecting duct system, renal pelvis, ureter, and bladder are histologically very similar, our data suggest that they are of distinct developmental origin. The SMCs of the inner medulla of the kidney that surround the collecting ducts and renal pelvis probably arise from common precursor cells of the stromal and nephrogenic mesenchymal cell lineages (20). The ureteral mesenchyme originates from the Tbx18positive zone abutting the metanephric mesenchyme in the early E11.5 kidney rudiment. The developmental origin of the SM layer of the bladder remains to be determined.

It is unclear how the condensation of ureteral cells around the distal ureteric epithelium occurs. Mesenchymal cells may actively migrate towards the epithelium, possibly guided by an epithelial chemoattractant. Alternatively, the mesenchymal cells may be pulled along passively, with elongation of the ureteric bud due to their preferential adhesion amongst themselves and to the ureteric epithelium. In any case, it seems clear that in the $T b \times 18^{-/-}$kidney, prospective ureteral cells fail to aggregate, and cover adjacent tissues instead. Most likely, mutant cells are pulled along passively with the growing and ascending kidney, since they preferentially cover the kidney surface closest to their initial position at E11.5. Dislocalization of $T b \times 18^{-/-}$cells may indicate a primary requirement for Tbx18 in promoting aggregation of mesenchymal cells that have been specified to become a ureteral subtype. Further defects, like reduction of cell proliferation and failure to differentiate into SMCs, may be caused by incompetence to perceive signals from the ureteric epithelium in conjunction with the lack of autocrine signaling due to reduced cell number and/or density. Alternatively, it may indicate a defect secondary to the loss of the ureteral specification of these precursor cells. It is difficult to distinguish these 2 possibilities since they actually may act in combination. In addition, the reciprocal signaling between ureteric epithelium and mesenchyme may preclude the identification of the primary defect. However, a role for Tbx18 in specifying ureteral cells is supported by the following evidence: expression of markers for ureteral mesenchyme including Sfrp2 was lost from the $T b \times 18^{-/-}$kidney before mesenchymal aggregation commenced, 
and $T b \times 18^{-/-}$cells that are retained around the ureteric epithelium failed to express markers for ureteral mesenchyme and did not differentiate into SMCs. Remarkably, Patched1 expression was downregulated from the ureteral mesenchyme despite normal Shb expression in the adjacent ureteric epithelium. Since Patched 1 is known to be a target of Shh in the kidney (22), this may suggest that the cells have not been specified and thus are not competent to respond to Shh signaling.

Tbx18 and SMC differentiation in the ureter. Ureteral mesenchymal cells can adopt at least 2 fates, probably correlating with their position relative to the ureteric epithelium. Mesenchymal cells of the inner compartment condense, proliferate more rapidly, and differentiate into SMCs. In contrast, mesenchymal cells of the outer ring remain loosely associated, show lower proliferation, and differentiate into fibrocytes of the adventitia. The $T b \times 18^{-/-}$ureter lacks SMCs, suggesting that patterning of the 2 mesenchymal compartments has failed. Both mesenchymal BMP4 and epithelial Shh signaling have been implicated in the differentiation of ureteral mesenchyme into SMCs. BMP4 is a member of TGF $\beta$ signaling family with multiple biological functions in metanephric kidney development, including the stimulation of ureter growth and elongation and a role as a chemoattractant for ureteral mesenchymal cells. In addition, BMP4 can induce SMC differentiation $(22,23$, $33,34)$. Bmp4 expression was absent from the mutant ureteral mesenchyme at E12.5, suggesting that the mutant mesenchyme is probably not responsive to Shh signaling, which would normally activate $B m p 4$ expression in this domain. This hypothesis is compatible with the observed downregulation of the Shh receptor Patched 1 in the Tbx18 mutant ureter. It should be noted that Bmp4 was still expressed in the mesenchyme surrounding the Wolffian duct at levels sufficient to assure its function in controlling the correct budding site of the ureter (data not shown).

It seems likely that the loss of BMP4 and Shh signaling contributes to the defects in mesenchymal aggregation and proliferation and SM differentiation in the Tbx18 mutant ureter. Mice with a conditional deletion of $S h b$ in the ureteric epithelium show reduced cell proliferation of undifferentiated mesenchymal cells and disturbed SM development. However, reduction of the SM layer of the ureter is much less severe than in $T b \times 18^{-/-}$mice, challenging the possibility that $T b x 18$ exclusively acts by conferring Shh responsiveness to the ureteral mesenchyme.

Loss of the angiotensin type I receptor (Agtr 1 ) gene and the gene of the potential downstream effector calcineurinB1 ( $C n b 1)$ both result in a reduction of SMC proliferation in the ureter and the pelvis. Agtr1 and Cnb1 mutant mice develop progressive renal obstruction due to a failure of the pyeloureteral peristaltic machinery (35, 36). Again, the phenotypic differences between Tbx18 and Agtr1 and $C n b 1$ mutant mice suggest a downstream role of angiotensin signaling in the expansion and maintenance of the SMC layers of the ureter rather than in the initial recruitment and differentiation of ureteral mesenchymal precursor cells.

Epithelial-mesenchymal interactions in ureter development. Reciprocal tissue interactions are a common theme in vertebrate organogenesis. Study of these interactions was initially focused on the metanephric kidney, where separation and recombination experiments revealed that both branching morphogenesis of the ureteric epithelium and nephron induction in the surrounding metanephric mesenchyme rely on signals from the adjacent tissue $(6,37)$. Development of the distal ureter and its surrounding ureteral mesenchymal layer lacks the complex morphogenetic processes of the proximal part. Nonetheless it is obvious that proliferation rates and differentiation waves require spatial and temporal coordination between the epithelium and the surrounding mesenchyme to achieve continuous elongation of the ureter and to acquire functional integrity at the onset of urine production in the kidney. Indeed, tissue recombination experiments have shown that the urothelium is necessary for the survival of isolated ureteral mesenchyme and induction of SMCs (38). These functions may be mediated at least in part by induction of $B m p 4$ expression through urothelial Shh signaling (22).

Our data suggest that signaling from the mesenchyme to the epithelium also occurs in ureter development. Loss of Tbx18 resulted in progressive reduction of proliferation rates not only in the ureteral mesenchyme, but also in the urothelium, leading to a short ureter at birth. In addition, the ureteric epithelium failed to differentiate into a functional urothelium, as shown by the lack of uroplakin expression. Hence epithelial cells may perceive signals from ureteral mesenchyme that adjust the proliferation rates and differentiation waves of both compartments. The molecular identity of these signals is unknown, but it seems possible that mesenchymal signaling depends on the number, density, and differentiation of ureteral mesenchymal cells.

The failure of urothelial differentiation may also contribute to the severe hydroureter and hydronephrosis phenotype of Tbx $18^{-/-}$ pups. Mice deficient for genes encoding components of the urothelial uroplakin plaques Uroplakin2 and Uroplakin3 experienced vesicoureteral reflux, hydronephrosis, and renal dysfunction, probably due to urothelial leakage and enlarged ureteral orifices $(39,40)$. However, urinary tract defects manifested primarily during postnatal development in these knockout animals, which suggests that the defects of mesenchymal development, particularly the lack of ureteral SMC differentiation, may be pivotal to the embryonic onset of hydroureter in $\mathrm{Tb} \times 18^{-/-}$mice.

Wnt signaling has been implicated in the control of cellular proliferation and differentiation processes in diverse programs during development (41). Expression of Wnt7b in the epithelium of collecting ducts and distal ureter and of $S f r p 2$ in ureteral mesenchyme may imply a role for Wnt signaling in distal ureter development $(24,25,28)$. Wnt7b regulates mesenchymal proliferation and vascular SM development in the lung (42). sFRP2, which belongs to the family of secreted frizzled related proteins, has been shown to modulate Wnt signaling during nephrogenesis in the metanephric kidney (43). Hence, sFRP2 in the ureteral mesenchyme may modulate a Wnt signal from the ureteric epithelium (e.g., Wnt7b) and from the ureteral mesenchyme. Loss of Sfrp2 in $T b \times 18^{-/-}$ureteral cells may lead to altered Wnt signaling and may contribute (in an unknown manner) to the various defects in the Tbx18 mutant ureter. It has been shown that notochord or recombinant Shh protein has the ability to upregulate the expression of Sfrp2 in the somitic mesoderm (44). Since the Shh signaling pathway also operates in ureter development (22), Sfrp2 expression in the ureteral mesenchyme may be regulated by Shh signals from the ureteric epithelium. However, early loss of Sfrp2 expression in mesenchymal cells relatively distant from the source of the Shh signal in the Tbx18 mutant may lend support to Shh-independent activation of Sfrp2 expression in this domain.

Tbx18 and bydroureter. Mice mutant for Tbx18 developed severe hydroureter, indicating an impaired transport of urine from the renal pelvis to the bladder. Hydronephrosis is a likely secondary consequence of hydroureter, as hydroureter manifested first and 
back pressure from the fluid-filled ureter is thought to dilate the renal pelvis. Fluid pressure then in turn causes the destruction of the renal parenchyme (4).

We failed to detect ectopic ureteric budding, multiplex kidneys, and nonseparation of ureter and Wolffian duct, which clearly excludes these well-known developmental defects (45-47) as causes or contributing factors to hydroureter formation in $T b \times 18$ mutant kidneys. As the dilatation of the ureter was observed along its entire length, the defect may arise from a ureterovesical junction abnormality. Analysis of ureter morphology in $T b \times 18^{-/-}$newborn mice detected a continuous lumen along the entire ureter length in few cases, whereas partial physical obstruction was more frequently observed. However, physical obstruction more likely represents a failure to expand the collapsed ureter lumen after relief of the hydrostatic pressure rather than a true structural obstruction of the ureter. It is very likely that the severe reduction or absence of ureteral SMCs is the major cause of hydroureter in Tbx $18^{-/-}$mice. SMCs mediate the peristaltic movements propelling the urine from the renal pelvis to the bladder. Lack of SMCs in the $T b \times 18^{-/-}$ureter may not only compromise the transport of urine, it may also fail to provide any mechanical rigidity to the tube to withstand the pressure of the fluid within.

Lack or scarcity of SMCs around the ureter is also associated with congenital human anomalies of the ureter as well as with mouse mutants generated by targeted gene disruption approaches $(20,48)$. In the human prune-belly syndrome the ureter shows great turtuosity and widening; the ureteral orifices appear widened and are abnormally located on the bladder. This is caused by a general hypoplasia of abdominal wall muscles including that of the ureter (49). In human congenital ureteral strictures, local reduction of SMCs leads to hydroureter proximal to the constriction $(5,7)$. In $S h b$ mutant mice, hydroureter develops due to a partial reduction of the SM layer of the renal pelvis and the ureter (22). Conditional removal of calcineurin function from the ureteral mesenchyme in the developing mouse embryo results in reduced proliferation of SMCs and ureteropelvic junction obstruction (36). In all of these examples onset and severity of hydroureter was less severe than in $T b \times 18^{-/-}$mice. This confirms that the complete lack of the SM coating of the ureter leads to an immediate dilatation of the ureter and renal pelvis with subsequent fluid pressure-mediated destruction of the renal parenchyme. Hence to our knowledge loss of $T b \times 18$ leads to the most severe case of hydroureter/hydronephrosis caused by a functional (and partially physical) ureter obstruction known to date.

To date, malformations of the urogenital system have not been associated with mutations in T-box genes. Thus, phenotypic characterization of $\mathrm{Tb} \times 18^{-/-}$mice has uncovered the first example to our knowledge of a Tbx gene involved in the etiology of anomalies of the kidneys and the urinary tract. Although association of mutations in human TBX18 with urinary tract defects has not been reported, the Tbx18 mouse may serve as a useful animal model to understand the etiology of hydroureter and hydronephrosis and malformation of SM coatings of epithelial tubes and to develop treatments for these diseases in humans.

\section{Methods}

Mice. Generation and genotyping of Tbx 18 heterozygous mice was recently described (14). We used the Tbx18Aneo allele, in which a phosphoglycerate kinase-neomycin (PGK.neo) resistance cassette was removed by cre-mediated recombination between loxP sites to allow $L a c Z$ expression from the
Tbx18 locus (Tbx18 $\left.{ }^{\text {LacZ }}\right)$, for most experiments. The unrecombined allele ( $T b x 18$ ) was used to normalize $L a c Z$ expression from the Tbx 18 locus. Tbx18 heterozygotes were maintained on an NMRI (Charles River) outbred background (14). Pax2:LacZ mice were kept on an NMRI outbred background (15). Embryos for kidney dissections were derived from matings of NMRI wild-type animals or Tbx18 heterozygotes. Wild-type or heterozygous littermates were used as controls for mutant embryos. H. Hedrich, state head of the animal facility at Medizinische Hochschule Hannover, approved the care of animals and experiments.

Collection of embryos. For timed pregnancies, plugs were checked in the morning after mating. Noon was designated E0.5. Embryos were dissected in PBS. Kidneys were isolated and fixed in $4 \%$ paraformaldehyde/PBS overnight, dehydrated in methanol, and stored at $-20^{\circ} \mathrm{C}$.

Ink injection experiments. To visualize the ureteropelvic lumen, Royal Blue ink (Pelikan) solution was injected into the pelvic region of kidneys of isolated whole urogenital systems using a pulled-out Pasteur glass pipette. Hydrostatic pressure was then applied to push the ink though the ureter to the bladder.

Kidney cultures. Cultures of E11.5 metanephric rudiments were performed as described previously (28). Rudiments were grown for 2 days before they were subjected to whole-mount histochemistry for $\beta$-galactosidase activity.

Histological analyses. Kidneys for histological stainings were fixed in $4 \%$ paraformaldehyde or in Bouin's fixative, paraffin embedded, and sectioned to 5 or $10 \mu \mathrm{m}$. Sections were stained with hematoxylin and eosin. Histochemistry for $\beta$-galactosidase activity was carried out as described previously (50). X-gal-stained urogenital systems were paraffin embedded and sectioned to $10 \mu \mathrm{m}$. Immunohistochemical detection of $\alpha$-SMA was performed on paraffin sections of Bouin's-fixed kidneys. Epitopes were recovered by boiling in Antigen Unmasking Solution (Vector Laboratories) for 15 minutes. The primary antibody (anti- $\alpha$-SMA, clone $1 \mathrm{~A} 4$; NatuTec) was applied overnight at $4{ }^{\circ} \mathrm{C}$ at a dilution of $1: 100$. The secondary antibody was goat anti-mouse IgG-HRP (Santa Cruz Biotechnology Inc.) at a dilution of 1:200. Color development relied on diaminobenzidine and $\mathrm{H}_{2} \mathrm{O}_{2}$ as substrates. Immunohistochemical detection of all 4 uroplakins on paraffin sections of E18.5 kidneys and ureters was performed using the avidin-biotin-peroxidase method similar to previously published procedures (51). The rabbit antiserum against total bovine uroplakins (a kind gift of T.-T. Sun, New York University, New York, New York, USA) was used in a dilution of 1:2,000 (30).

Proliferation and apoptosis assays. Cell proliferation in the ureters of E12.5 and E15.5 embryos was investigated by detection of incorporated BrdU on 5 - $\mu \mathrm{m}$ sections of paraffin-embedded specimens similar to previously published protocols (14). Ten sections each of 4 embryos of each genotype at E12.5 and 20 sections each of 4 embryos of each genotype at E15.5 were used for quantification. The BrdU-labeling index was defined as the number of BrdU-positive nuclei relative to the total number of nuclei, as detected by DAPI counterstain, in the ureter region. Detection of apoptotic cells in 10$\mu \mathrm{m}$ paraffin sections of E12.5 and E15.5 embryos was based on modification of genomic DNA utilizing terminal deoxynucleotidyl transferase (TUNEL assay) and indirect detection of positive cells by fluorescein-conjugated antidigoxigenin antibody. The procedure followed exactly the recommendation of the manufacturer (Serologicals Corp.) of the ApopTag kit used.

In situ hybridization analysis. Whole-mount in situ hybridization was performed following a standard procedure with digoxigenin-labeled antisense riboprobes (52). Stained specimens were transferred in $80 \%$ glycerol prior to documentation. In situ hybridization analysis on $10-\mu \mathrm{m}$ paraffin sections was done essentially as described previously (25).

Documentation. Whole-mount specimens were photographed on a Leica M420 microscope with the Fujix digital camera HC-300Z. Sections were photographed on a Leica Axioplan microscope with a ProgResC14 digital camera. All images were processed in Adobe Photoshop version 7.0. 
Statistics. Statistical analyses were performed using the 2-tailed Student's $t$ test. Data were expressed as mean \pm SEM. Differences were considered significant when the $P$ value was below 0.05 .

\section{Acknowledgments}

We thank Frank Constanini, Suzanne Cory, Ahmed Mansouri, Cathy Mendelsohn, Matthew Scott, Liz Robertson, and Andrew P. McMahon for probes; Tung-Tien Sun for the rabbit antiserum against total bovine uroplakins; and Henner Farin and Achim Gossler for critical discussion. The Uroplakin3a cDNA was obtained from the Resourcenzentrum in Berlin, Germany. This work was supported by a grant from the Deutsche Forschungsgemeinschaft (DFG KI728/2) to A. Kispert.

Received for publication June 21, 2005, and accepted in revised form December 13, 2005.

Address correspondence to: Andreas Kispert, Institut für Molekularbiologie, OE5250, Medizinische Hochschule Hannover, Carl-Neuberg-Str. 1, 30625 Hannover, Germany. Phone: 49-511-5324017; Fax: 49-511-5324283; E-mail: kispert.andreas@ mh-hannover.de.
1. Kohaut, E.C., and Tejani, A. 1996.The 1994 annual report of the North American Pediatric Renal Transplant Cooperative Study. Pediatr. Nephrol. 10:422-434.

2. Woolf, A.S., and Winyard, P.J. 2002. Molecular mechanisms of human embryogenesis: developmental pathogenesis of renal tract malformations. Pediatr. Dev. Pathol. 5:108-129.

3. Miyazaki, Y., and Ichikawa, I. 2003. Ontogeny of congenital anomalies of the kidney and urinary tract, CAKUT. Pediatr. Int. 45:598-604.

4. Chevalier, R.L. 2004. Perinatal obstructive nephropathy. Semin. Perinatol. 28:124-131.

5. Tanagho, E.A. 1981. Development of the ureter. In The ureter. H. Bergman, editor. Springer-Verlag. New York, New York, USA. 1-12.

6. Saxen, L. 1987. Organogenesis of the kidney. Cambridge University Press. New York, New York, USA. 184 pp.

7. Culp, D.A. 1981. Congenital anomalies of the ureter. In The ureter. H. Bergman, editor. Springer-Verlag. New York, New York, USA. 625-646.

8. Mendelsohn, C. 2004. Functional obstruction: the renal pelvis rules. J. Clin. Invest. 113:957-959. doi:10.1172/JCI200421402.

9. Sariola, H., and Saarma, M. 1999. GDNF and its receptors in the regulation of the ureteric branching. Int. J. Dev. Biol. 43:413-418.

10. Naiche, L.A., Harrelson, Z., Kelly, R.G., and Papaioannou, V.E. 2005. T-box genes in vertebrate development. Annu. Rev. Genet. 39:219-239.

11. Tada, M., and Smith, J.C. 2001. T-targets: clues to understanding the functions of T-box proteins. Dev. Growth Differ. 43:1-11.

12. Packham, E.A., and Brook, J.D. 2003. T-box genes in human disorders. Hum. Mol. Genet. 12:R37-R44.

13. Kraus, F., Haenig, B., and Kispert, A. 2001. Cloning and expression analysis of the mouse T-box gene Tbx18. Mech. Dev. 100:83-86.

14. Bussen, M., et al. 2004. The T-box transcription factor Tbx18 maintains the separation of anterior and posterior somite compartments. Genes Dev. 18:1209-1221.

15. Kuschert, S., Rowitch, D.H., Haenig, B., McMahon, A.P., and Kispert, A. 2001. Pax-2 regulatory sequences that direct transgene expression in the Wolffian duct and its derivatives. Dev. Biol. 229:128-140.

16. Neidhardt, L.M., Kispert, A., and Herrmann, B.G. 1997. A mouse gene of the paired-related homeobox class expressed in the caudal somite compartment, and in the developing vertebral column, kidney and nervous system. Dev. Genes Evol. 207:330-339.

17. Armstrong, J.F., Pritchard-Jones, K., Bickmore, W.A., Hastie, N.D., and Bard, J.B. 1993. The expression of the Wilms' tumour gene, WT1, in the developing mammalian embryo. Mech. Dev. 40:85-97.

18. Hatini, V., Huh, S.O., Herzlinger, D., Soares, V.C., and Lai, E. 1996. Essential role of stromal mesenchyme in kidney morphogenesis revealed by targeted disruption of Winged Helix transcription factor BF-2. Genes Dev. 10:1467-1478.

19. Batourina, E., et al. 2001. Vitamin A controls epithelial/mesenchymal interactions through Ret expression. Nat. Genet. 27:74-78.

20. Levinson, R., and Mendelsohn, C. 2003. Stromal progenitors are important for patterning epithelial and mesenchymal cell types in the embryonic kidney. Semin. Cell Dev. Biol. 14:225-231.

21. Quaggin, S.E., et al. 1999. The basic-helix-loop-helix protein pod 1 is critically important for kidney and lung organogenesis. Development. 126:5771-5783.

22. Yu, J., Carroll, T.J., and McMahon, A.P. 2002. Sonic hedgehog regulates proliferation and differentiation of mesenchymal cells in the mouse metanephric kidney. Development. 129:5301-5312.

23. Miyazaki, Y., Oshima, K., Fogo, A., Hogan, B.L., and Ichikawa, I. 2000. Bone morphogenetic protein 4 regulates the budding site and elongation of the mouse ureter. J. Clin. Invest. 105:863-873.

24. Leimeister, C., Bach, A., and Gessler, M. 1998. Developmental expression patterns of mouse sFRP genes encoding members of the secreted frizzled related protein family. Mech. Dev. 75:29-42.

25. Lescher, B., Haenig, B., and Kispert, A. 1998. sFRP-2 is a target of the Wnt-4 signaling pathway in the developing metanephric kidney. Dev. Dyn. 213:440-451.

26. Pachnis, V., Mankoo, B., and Costantini, F. 1993. Expression of the c-ret proto-oncogene during mouse embryogenesis. Development. 119:1005-1017.

27. Miyamoto, N., Yoshida, M., Kuratani, S., Matsuo, I., and Aizawa, S. 1997. Defects of urogenital development in mice lacking Emx2. Development. 124:1653-1664.

28. Kispert, A., Vainio, S., Shen, L., Rowitch, D.H., and McMahon, A.P. 1996. Proteoglycans are required for maintenance of Wnt-11 expression in the ureter tips. Development. 122:3627-3637.

29. Sun, T.T., Liang, F.X., and Wu, X.R. 1999. Uroplakins as markers of urothelial differentiation. $A d v$. Exp. Med. Biol. 462:7-18; discussion 103-114.

30. Wu, X.R., Manabe, M., Yu, J., and Sun, T.T. 1990 Large scale purification and immunolocalization of bovine uroplakins I, II and III. Molecular markers of urothelial differentiation. J. Biol. Chem. 265:19170-19179.

31. Leitges, M., Neidhardt, L., Haenig, B., Herrmann, B.G., and Kispert, A. 2000. The paired homeobox gene $U n c x 4.1$ specifies pedicles, transverse processes and proximal ribs of the vertebral column. Development. 127:2259-2267.

32. Mansouri, A., Voss, A.K., Thomas, T., Yokota, Y., and Gruss, P. 2000. Uncx4.1 is required for the formation of the pedicles and proximal ribs and acts upstream of Pax9. Development. 127:2251-2258.

33. Raatikainen-Ahokas, A., Hytonen, M., Tenhunen, A., Sainio, K., and Sariola, H. 2000. BMP-4 affects the differentiation of metanephric mesenchyme and reveals an early anterior-posterior axis of the embryonic kidney. Dev. Dyn. 217:146-158.

34. Miyazaki, Y., Oshima, K., Fogo, A., and Ichikawa, I. 2003. Evidence that bone morphogenetic protein 4 has multiple biological functions during kidney and urinary tract development. Kidney Int. 63:835-844.

35. Miyazaki, Y., et al. 1998. Angiotensin induces the urinary peristaltic machinery during the perinatal period. J. Clin. Invest. 102:1489-1497.

36. Chang, C.-P., et al. 2004. Calcineurin is required in urinary tract mesenchyme for the development of the peyeloureteral peristaltic machinery. J. Clin. Invest. 113:1051-1058. doi:10.1172/JCI200420049.

37. Grobstein, C. 1953. Inductive epitheliomesenchymal interaction in cultured organ rudiments of the mouse. Science. 118:52-55.

38. Cunha, G.R. 1976. Epithelial-stromal interactions in development of the urogenital system. Int. Rev. Cytol. 47:137-194.

39. Hu, P., et al. 2000. Ablation of uroplakin III gene results in small urothelial plaques, urothelial leakage, and vesicoureteral reflux. J. Cell Biol. 151:961-972.

40. Kong, X.T., et al. 2004. Roles of uroplakins in plaque formation, umbrella cell enlargement, and urinary tract diseases. J. Cell Biol. 67:1195-1204.

41. Logan, C.Y., and Nusse, R. 2004. The Wnt signaling pathway in development and disease. Annu. Rev. Cell Dev. Biol. 20:781-810.

42. Shu, W., Jiang, Y.Q., Lu, M.M., and Morrisey, E.E. 2002. Wnt7b regulates mesenchymal proliferation and vascular development in the lung. Development. 129:4831-4842.

43. Yoshino, K., et al. 2001. Secreted Frizzled-related proteins can regulate metanephric development. Mech. Dev. 102:45-55.

44. Lee, C.S., Buttitta, L.A., May, N.R., Kispert, A., and Fan, C.-M. 2000. SHH-N upregulates sfrp2 to mediate its competitive interaction with WNT1 and WNT4 in the somitic mesoderm. Development. 127:109-118.

45. Kume, T., Deng, K., and Hogan, B.L. 2000. Murine forkhead/winged helix genes Foxc1 (Mf1) and Foxc2 (Mfh1) are required for the early organogenesis of the kidney and urinary tract. Development. 127:1387-1395

46. Batourina, E., et al. 2002. Distal ureter morphogenesis depends on epithelial cell remodeling mediated by vitamin A and Ret. Nat. Genet. 32:109-115.

47. Basson, M.A., et al. 2005. Sprouty1 Is a critical regulator of GDNF/RET-mediated kidney induction. Dev. Cell. 8:229-239.

48. Dure-Smith, P., Lau, L., Khan, B., and David, A. 2002. Congenital variations in mucomuscular development of the ureter. BJU Int. 90:130-134.

49. Jennings, R.W. 2000. Prune belly syndrome. Semin. Pediatr. Surg. 9:115-120.

50. Echelard, Y., Vassileva, G., and McMahon, A.P. 1994. Cis-acting regulatory sequences governing Wnt-1 expression in the developing mouse CNS. Development. 120:2213-2224.

51. Ogawa, K., Johansson, S.L., and Cohen, S.M. 1999. Immunohistochemical analysis of uroplakins, urothelial specific proteins, in ovarian Brenner tumors, normal tissues, and benign and neoplastic lesions of the female genital tract. Am. J. Pathol. 155:1047-1050.

52. Wilkinson, D.G. 1992. Whole mount in situ hybridization of vertebrate embryos. In In situ bybridization: a practical approach. D.G. Wilkinson, editor. Oxford University Press. Oxford, United Kingdom. 75-84. 